\title{
Preprints and catalysis
}

\author{
Preprints are openly available non-peer-reviewed manuscripts that precede the actual peer-reviewed publication. \\ We analyse this phenomenon that is gaining popularity across all areas of catalysis.
}

S: ome seek feedback before submission to a journal, some want to raise awareness or claim precedence of their upcoming publication, some may even look for alternatives beyond traditional publishing. There are many reasons for authors to upload their works to preprint servers, and it is undeniable that they are growing in popularity, within the catalysis community and beyond.

Preprints - or, as they are also called, e-prints - have their origin in the arXiv repository, which started in 1991 in Los Alamos National Laboratory in New Mexico, and was a decade later transferred to Cornell University in Washington DC. Initially intended to host studies across all areas of physics, arXiv soon expanded to other fields such as mathematics, computer science, quantitative biology and, more recently, economics. Subsequently, other servers appeared, including bioRxiv, PeerJ Preprints, engrXiv or the very recent ChemRxiv, hosting preprints across all life and physical sciences. Their popularity has skyrocketed in recent years, especially after the advent of these subject-specific archives that truly sparked widespread uptake outside the physics community. For instance, the platform PrePubMed (http://www. prepubmed.org/), which gathers and links life science preprints from nine different servers, tracks the number of files uploaded in this area. These have increased from 100 per month in 2009 to over 2,000 per month in 2018, due in particular to the success of bioRxiv.

The first preprint on the subject of catalysis dates from 1995 and was deposited on $\operatorname{arXiv}^{1}$. Although some areas of catalysis fall within the scope of arXiv, bioRxiv, or more general preprint servers such as preprints.org, it is clear that catalysis and the recent chemistry-specific archives, such as ChemRxiv or ChemRN, have greatly benefited from each other. A search in ChemRxiv for the words catalysis, catalyst or catalytic in the title, description or category, returned almost $20 \%$ of the total content. And indeed, we have recently published our first paper that had originally been deposited on a preprint repository, this being ChemRxiv ${ }^{2}$. This server, which was launched in August 2017, is progressively gaining popularity and becoming an important resource for the chemistry and catalysis communities. The scope of arXiv certainly covers heterogeneous, electro and photocatalysis, and bioRxiv does host works related to biocatalysis, but the overlap between the chemistry-specific repositories and catalysis is complete.

Scientists, however, have not yet adopted preprint archival as a mainstream practice, especially outside the physics community. But while many researchers are either unfamiliar or uncertain about them, preprints have already begun to take root within the chemistry and catalysis communities. "The biggest issues were around confusion about what preprints are for and whether journals would take them", says Marshall Brennan, the Publishing Manager of ChemRxiv, about the beginnings of this platform. "Through outreach and education, we managed to get enough authors to come on board that the journals that initially resisted preprints changed their stance", he adds. Now the server hosts over 1,100 preprints, with almost a million views and 200,000 downloads, which have substantially exceeded their expectations. Readers "have never been hard to convince".

\section{"Our policy at Nature Catalysis is that we do not consider pre- prints to compromise the nov- elty of a given submitted work."}

Journals do not unanimously support the use of preprints yet. According to SHERPA/ RoMEO (http://www.sherpa.ac.uk/romeo/ index.php), $18 \%$ of all publishers do not allow manuscript archiving in the form of preprint or postprint. This high percentage does not reflect, however, the catalysis publishing community, where practically all journals consider manuscripts that have been previously uploaded as preprints.
Our policy at Nature Catalysis is that we do not consider preprints to compromise the novelty of any given submitted work. We therefore support posting originally submitted versions of manuscripts on preprint servers, which may be posted at any time. This is a common policy for all Nature Research journals (https://www.nature.com/ authors/policies/preprints.html) and dates back to an editorial published in Nature in 1997 (ref. ${ }^{3}$ ). We also ask our authors to respect our policy on communications with the media (https://www.nature.com/authors/ policies/embargo.html).

Nature Communications started last year an initiative where authors of submitted manuscripts can list their preprint with a link to the archive file if the article is sent out for peer-review (https://natureresearch-under-consideration.nature.com/). The preprint is then removed from the list after the submitted manuscript is either accepted or rejected from the journal. After over a year of trial, the initiative has been a success and many authors have opted to link the preprint to their manuscript under consideration. Similarly, the recently launched Nature Machine Intelligence allows authors to add a link to the preprint version alongside the published article.

The communities of the various areas of catalysis have different paces, and while some of those working on heterogeneous catalysis have been uploading preprints for more than two decades, their use among those working on other areas of catalysis started much more recently. We think that the benefits of preprints are manifold and thus we expect them to consolidate in all areas of catalysis in the not too distant future.

Published online: 13 February 2019 https://doi.org/10.1038/s41929-019-0243-5

\footnotetext{
References

1. Frachebourg, L., Krapivsky, P. L. \& Redner, S. Preprint at https://arxiv.org/abs/cond-mat/9506122 (1995).

2. Lichosyt, D., Zhang, Y., Hurej, K. \& Dydio, P. Preprint at https://doi.org/10.26434/chemrxiv.7235438.vl (2018).

3. Nature 390, 427 (1997)
} 